\title{
AGR2, a mucinous ovarian cancer marker, promotes cell proliferation and migration
}

\author{
Kyoungsook Park', Yong Jin Chung ${ }^{1}$, \\ Hyekyung So ${ }^{1}$, Kwangsoo Kim ${ }^{1}$, Junsoo Park', \\ Mijoung Oh ${ }^{1}$, Minwha Jo ${ }^{1}$, Kyusam Choi ${ }^{1}$, \\ Eun-Ju Lee ${ }^{5}$, Yoon-La Choi ${ }^{2}$, Sang Yong Song', \\ Duk-Soo Bae ${ }^{3}$, Byoung-Gie $\mathrm{Kim}^{3}$ and Je-Ho Lee ${ }^{2,6}$ \\ ${ }^{1}$ Samsung Biomedical Research Institute \\ ${ }^{2}$ Department of Pathology \\ ${ }^{3}$ Department of Obstetrics and Gynecology \\ Samsung Medical Center \\ Sungkyunkwan University School of Medicine \\ Seoul 135-710, Korea \\ ${ }^{4}$ Division of Biological Science and Technology \\ Yonsei University \\ Wonju 220-710, Korea \\ ${ }^{5}$ Department of Obstetrics and Gynecology \\ Chung-Ang University School of Medicine \\ Seoul 156-755, Korea \\ ${ }^{6}$ Corresponding author: Tel, 82-2-3410-3510; \\ Fax, 82-2-3410-4400; E-mail, jeholee@ gmail.com \\ DOI 10.3858/emm.2011.43.2.011
}

Accepted 22 December 2010

Available Online 4 January 2011

Abbreviations: AGR2, anterior gradient 2; agt, angiotensinogen; RT-CES, real-time cell electronic sensing system; THBS1, thrombospondin 1 ; vegf $A$, vascular endothelial growth factor $A$

\begin{abstract}
Ovarian cancer is a leading cause of death in women. Early detection of ovarian cancer is essential to decrease mortality. However, the early diagnosis of ovarian cancer is difficult due to a lack of clinical symptoms and suitable molecular diagnostic markers. Thus, identification of meaningful tumor biomarkers with potential clinical application is clearly needed. To search for a biomarker for the early detection of ovarian cancer, we identified human anterior gradient 2 (AGR2) from our systematic analysis of paired normal and ovarian tumor tissue cDNA microarray. We noted a marked overexpression of AGR2 mRNA and protein in early stage mucinous ovarian tumors compared to normal ovarian tissues and serous type ovarian tumors by Western blot analysis and immunohistochemistry. To further elucidate the role of AGR2 in ovarian tumori-
\end{abstract}

genesis, stable 2774 human ovarian cancer cell lines overexpressing AGR2 were established. Forced expression of AGR2 in $\mathbf{2 7 7 4}$ cells enhanced the growth and migration of ovarian cancer cells. AGR2 protein was detected in the serum of mucinous ovarian cancer patients by Western blot and ELISA analysis. Thus, AGR2 is a potential biomarker for the diagnosis of mucinous ovarian cancer and an ELISA assay may facilitate the early detection of mucinous ovarian cancer using patient serum.

Keywords: AGR2 protein, human; biological markers; ovarian neoplasms

\section{Introduction}

Ovarian cancer is one of the most lethal gynecologic malignancies and has among the worst of prognoses. The lethality of ovarian cancer is primarily attributable to the advanced stage of the disease at the time of the initial diagnosis. Although the cure rate from currently available therapies is as high as $90 \%$ when the cancer is detected in an early stage (American Cancer Society, 2005), the majority of early-stage cancers are asymptomatic and most cases are diagnosed at an advanced stage. Thus, long-term survival can be achieved in $<30 \%$ of patients (Edwards et al., 2005). Therefore, screening for an early-stage diagnosis can significantly reduce mortality. The identification of reliable diagnostic biomarkers for the early detection of ovarian cancer is critical for reducing the mortality rate of ovarian cancer. Furthermore, identification of meaningful tumor biomarkers with potential clinical application would provide useful information regarding patient diagnosis, prognosis, and possible therapeutic options.

Anterior gradient homolog 2 (AGR2), also known as hAG-2 (Fletcher et al., 2003) and Gob-4 (Komiya et al., 1999), is the human orthologue of the secreted Xenopus laevis anterior gradient protein, XAG-2. XAG-2 has a putative role in ectodermal patterning of the frog embryo and is regulated by noggin and chordin (Aberger et al., 1998). The expression of XAG-2 induces cement gland differentiation and expression of neural marker genes in a fibroblast growth factor-dependent manner (Aberger et al., 1998). Up-regulation of the AGR2 gene has been reported in breast cancer (Fri- 
tzsche et al., 2006), prostate cancer (Kristiansen et al., 2005; Zhang et al., 2005), and endometrial and renal carcinomas (Fritzsche et al., 2006). AGR2 has been proposed as one of the potential markers to detect circulating tumor cells in the peripheral blood in patients with metastatic cancers, including colorectal, prostate, and breast cancers (Smirnov et al., 2005). The expression of AGR2 has also been described to be associated with a positive estrogen receptor status of tumor cells. However, the expression and role of AGR2 in mucinous ovarian cancer remains uncharacterized and requires further study.

Initially, we set out to identify potential biomarkers that could be used as a non-invasive method of early diagnosis of ovarian cancer by cDNA microarray analysis. Herein we report that the secreted protein, AGR2, is highly overexpressed in human mucinous ovarian tumor tissues and patient sera compared with tissue and sera samples from healthy women, as determined by biochemical and molecular approaches. To further explore the roles of AGR2 in ovarian tumorigenesis, stable AGR2-overexpressing human ovarian cancer cells were established and characterized. The findings from this study suggest that AGR2 can serve as a potential diagnostic marker for the detection of mucinous ovarian cancer and AGR2 detection may provide an efficient and sensitive tool for the early detection of human mucinous ovarian cancer.

\section{Results}

\section{Identification of a novel biomarker for human mucinous ovarian cancer}

To identify novel molecular markers that are more sensitive and specific for the early detection of ovarian cancer, cDNA microarray was carried out and analyzed with 32 tissue samples from ovarian cancer patients. Among the up-regulated genes, AGR2 was chosen because the subcellular localization of AGR2 using the PSORT II program revealed that it is a secretory protein. AGR2 was markedly up-regulated in all mucinous-type ovarian cancers, but no significant expression $(<25 \%$ of all cases) was observed in serous-type ovarian cancers compared with normal ovarian tissues (Figure 1). A scatter plot shows a $>5$-fold upregulation of AGR2 mRNA in mucinous ovarian tumors, whereas the majority of serous-type ovarian tumors had a $<5$-fold up-regulation (Figure $1 \mathrm{~A})$. A further comparison between adenocarcinoma and borderline tumors in each tumor type clearly revealed preferential expression of
A

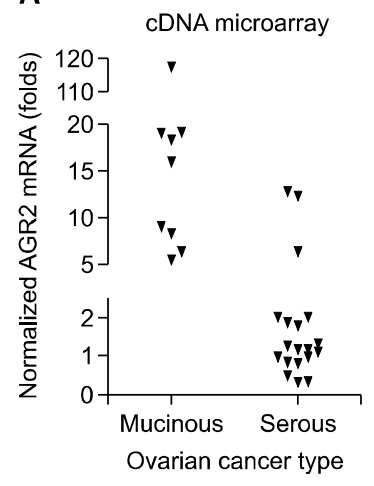

B

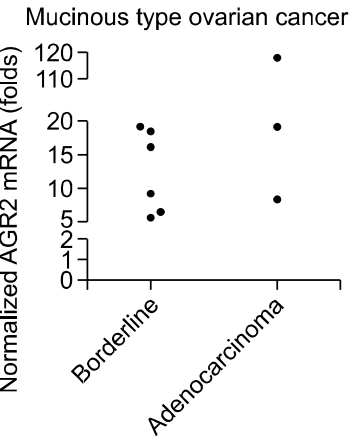

Serous type ovarian cancer
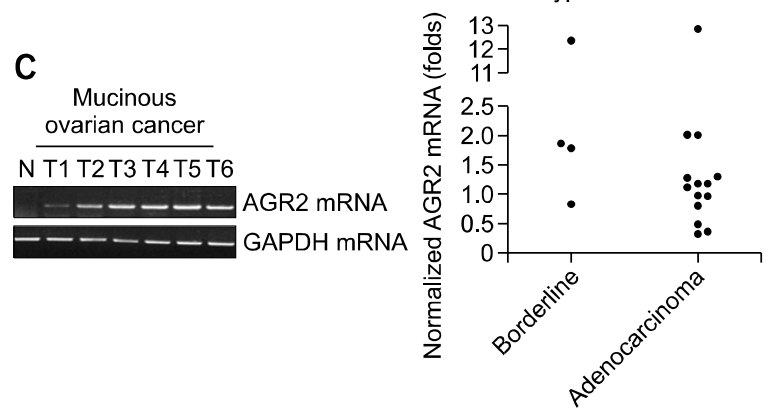

Figure 1. Up-regulation of $A G R 2$ mRNA in human mucinous ovarian cancer. (A, B) Scatter plot analysis of cDNA microarray. AGR2 expression was analyzed according to the tumor type $(A)$ and cancer grade (B). Scattered plot shows > 5-fold up-regulation of AGR2 mRNA in mucinous ovarian tumors, whereas the majority of serous-type ovarian tumors had a $<5$-fold up-regulation. (C) RT-PCR analysis of AGR2 expression in mucinous ovarian cancer tissues. Total RNA was used to confirm the up-regulation of AGR2 by CDNA microarray in mucinous ovarian tumor tissues. GAPDH mRNA was used as a control to normalize the level of mRNA in each sample. N: normal ovary.

AGR2 in mucinous ovarian tumors compared with serous ovarian tumors (Figure 1B). Next, to confirm the expression of AGR2 mRNA in mucinous ovarian cancers, RT-PCR analysis was performed in six different human mucinous ovarian cancer tissues and one normal human ovarian tissue (Figure 1C). In agreement with microarray data, human mucinous cancer had a markedly increased expression of AGR2 mRNA.

To determine the level of expression of AGR2 protein, 20 ovarian tumors and 4 normal healthy ovarian tissues were immunostained with antiAGR2 antibody (Figure 2A). AGR2 protein was detected in the cytoplasm of all tissues. AGR2 protein was expressed at a basal level in normal ovary surface epithelium (lane 1) and serous ovarian tumor tissues (lanes 4-5), whereas a highly elevated level of AGR2 protein expression was detected in mucinous ovarian tumors (lanes 2-3; Figure 2B). These results suggest that AGR2 protein may be a good marker for the detection of mucinous ovarian tumors. AGR2 protein ex- 
A

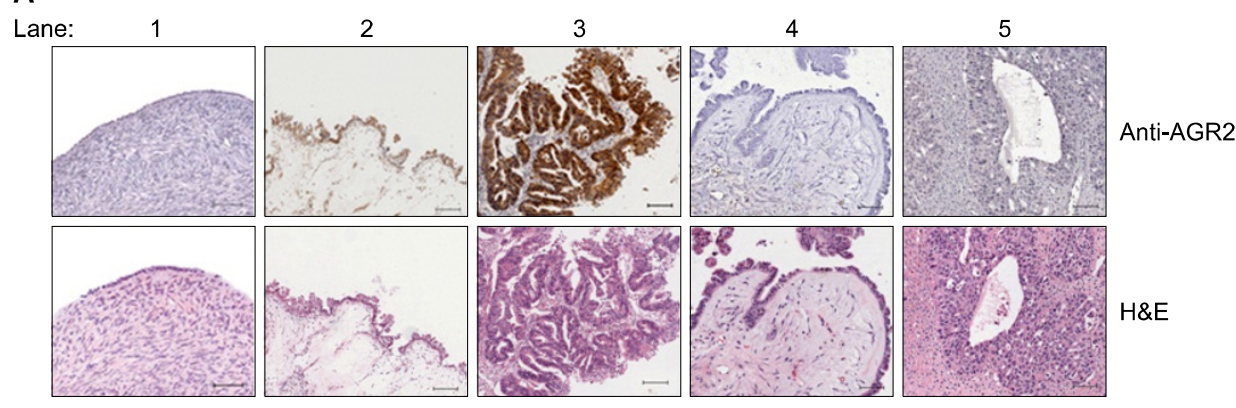

B

C

\begin{tabular}{|c|c|c|}
\hline $\begin{array}{c}\text { Type of } \\
\text { ovarian } \\
\text { cancer }\end{array}$ & Cancer grade & $\begin{array}{c}\text { Stained samples } \\
\text { /total samples }\end{array}$ \\
\hline \multirow{2}{*}{$\begin{array}{c}\text { Mucinous } \\
\text { type }\end{array}$} & Borderline & $3 / 3$ \\
\cline { 2 - 3 } & Adenocarcinoma & $4 / 4$ \\
\cline { 2 - 3 } & Total & $7 / 7$ \\
\hline \multirow{2}{*}{$\begin{array}{c}\text { Serous } \\
\text { type }\end{array}$} & Borderline & $0 / 1$ \\
\cline { 2 - 3 } & Adenocarcinoma & $6 / 12$ \\
\cline { 2 - 3 } & Total & $6 / 13$ \\
\hline
\end{tabular}

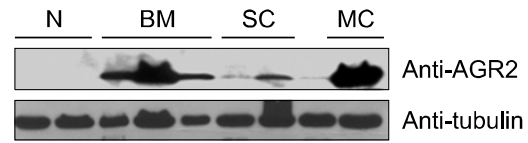

Figure 2. Overexpression of AGR2 protein in human mucinous ovarian cancer. (A) Top panels: Immunohistochemistry of AGR2 protein in various types of ovarian tumor tissues. The sections were counterstained with hematoxylin as follows: 1) normal ovary; 2) borderline mucinous tumor of the ovary; 3) mucinous adenocarcinoma of the ovary; 4) borderline serous tumor of the ovary; and 5) serous adenocarcinoma of the ovary. Bottom panels: Corresponding H\&E staining of respective tumor section (Scale bar: $50 \mu \mathrm{m}$ ). (B) Summary of AGR2 protein expression in ovarian cancer by immunohistochemistry. AGR2 expression was further analyzed according to the tumor type and cancer grade. (C) Expression of AGR2 protein in ovarian tumor by Western blot analysis. Total tissue lysates were prepared in RIPA buffer and then subjected to Western blot analysis with anti-AGR2 antibody. N, Normal ovary; BM, Borderline mucinous tumor of the ovary; SC, Serous adenocarcinoma of the ovary; MC, Mucinous adenocarcinoma of the ovary.

pression in ovarian tumor tissues was further confirmed by Western blot analysis (Figure 2C). The anti-AGR2 antibody detected a predominant $18 \mathrm{kDa}$ protein in the protein extracts from mucinous ovarian tumors. In contrast, there was weak or no detectable AGR2 protein in normal ovarian tissues. These results are consistent with immunohistochemical staining of AGR2 protein, and very high expression of AGR2 protein in mucinous ovarian tumors compared with no staining in normal counterparts. These results confirmed the markedly elevated AGR2 expression of protein in mucinous ovarian tumor tissues.

\section{Generation of human ovarian cancer cells overexpressing human AGR2}

To further elucidate the role and mechanism of AGR2 in ovarian tumorigenesis, human AGR2overexpressing ovarian cancer cells were generated by G418 selection (Figure $3 A$ ). Control vector- and AGR2-expressing stable cells were tested for the expression of AGR2 protein by
Western blot analysis and confocal microscopy after indirect immunofluorescence staining (Figure 3B). Compared to control vector stable transfectants, AGR2 stable transfectants (clones A1 and A2) exhibited expression of AGR2 protein. AGR2 stable transfectants showed strong cytoplasmic staining of AGR2 protein by confocal imaging analysis, whereas control vector stable cells had no staining for AGR2 protein (Figure 3B). The subcellular localization of AGR2 protein was predicted to be cytoplasmic and secreted using the PSORT II program (http://psort.nibb.ac.jp). To clarify the subcellular localization of overexpressed AGR2 protein, 2774-AGR2 stable transfectants were biochemically fractionated, then AGR2 protein expression was assessed by Western blot analysis with anti-AGR2 antibody (Figure 3C). AGR2 protein was preferentially expressed in the cytoplasm. Furthermore, to confirm secretion of AGR2 protein in the serum-free conditioned medium, conditioned medium from the AGR2 stable transfectants were analyzed by Western blot analysis with anti-AGR2 antibody (Figure 3D). As 

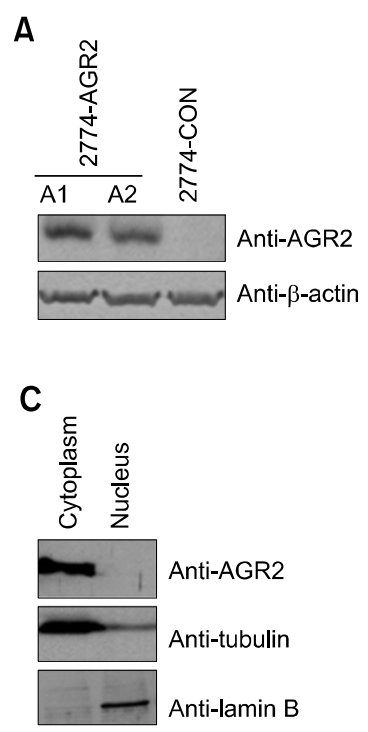

B

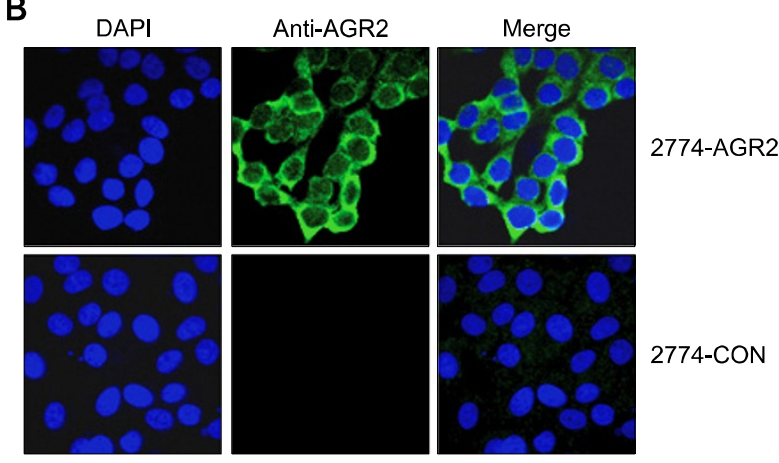

D

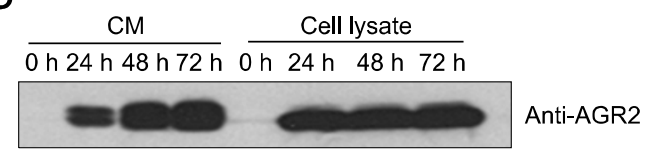

Figure 3. Establishment of AGR2 overexpressing human 2774 ovarian cancer stable transfectants. (A) Expression of AGR2 protein in 2774 stable transfectants. Human AGR2 mammalian expression vector was transfected into 2774 cells, then AGR2-overexpressing stable cells were selected by G418 selection. Stable 2774 transfectants transfectants were assessed for AGR2 protein expression by Western blot analysis with anti-AGR2 antibody. (B) Subcellular localization of AGR2 in AGR2 stable transfectants. Vector control and AGR2 stable transfectants were processed for indirect immunofluorescence microscopy for subcellular localization of AGR2 protein in human 2774 ovarian cancer cells. AGR2 protein was expressed in all AGR2 stable transfectants and showed a strong preferential cytoplasmic localization. (C) Subcellular localization of AGR2 protein. Whole cell lysates prepared in RIPA buffer from the stable 2774 cells were fractionated, then subjected to Western blot analysis with anti-AGR2 antibody. AGR2 protein was detected in the cytoplasmic fraction. Subcellular fractionation was confirmed by Western blot analysis with anti-lamin B and anti-tubulin antibodies. (D) AGR2 protein is a secreted protein. Conditioned medium was collected from the indicated stable AGR2 transfected 2774 cells at the indicated incubation time, then analyzed by Western blot analysis with anti-AGR2 antibody.

expected, we were able to detect AGR2 protein in the serum-free conditioned medium in AGR2 stable transfectants.

\section{Enhancement of ovarian cancer cell proliferation and migration by AGR2 overexpression}

To determine the effect of AGR2 overexpression in human ovarian cancer cells, AGR2-expressing stable 2774 ovarian cancer cells were tested for the effect on cancer cell growth by a real-time cell electronic sensor system (CES). Forced expression of AGR2 increased ovarian cancer cell growth and proliferation (Figure 4A). Next, the effect of AGR2 on cancer cell migration was assessed using a Boyden chamber. Overexpression of AGR2 significantly increased cancer cell migration that is necessary for cancer cell invasion (Figure 4B).

To gain insight into the function of AGR2 in ovarian tumorigenesis, total RNA extracted from control vector and AGR2 stable cells was used for cDNA microarray analysis to identify the target genes for further functional studies. CDNA microarray analysis of AGR2 stable transfectants revealed that $A G R 2$ overexpression up-regulated the expression of genes involved in cell proliferation, invasion, and angiogenesis, which is important for tumor progression and metastasis. For example, expression of vascular endothelial growth factor $\mathrm{A}$ (vegf $A$ ) and angiotensinogen (agt) genes, which are secreted and involved in angiogenesis, were elevated 3.44- and 4.45-fold, respectively, whereas interleukin-8 (IL-8) and thrombospondin 1 (THBS1), which are involved in cell adhesion and negative regulation of cell proliferation, were each reduced 2-fold (Table 1). Furthermore, the genes involved in cell death and cell cycle regulation were down-regulated. The genes involved in cell adhesion, such as $P_{-}$ cadherin and THBS1, were down-regulated.

\section{Detection of AGR2 protein in the serum from ovarian cancer patients}

Serum samples were analyzed for the detection of AGR2 protein by Western blot analysis with antiAGR2 antibody (Figure 5A). Equal protein loading of serum samples was confirmed by Ponceau $S$ staining of the same blot (bottom panel). AGR2 protein expression was significantly elevated in the 
A

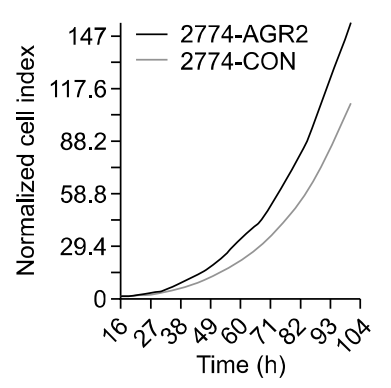

B

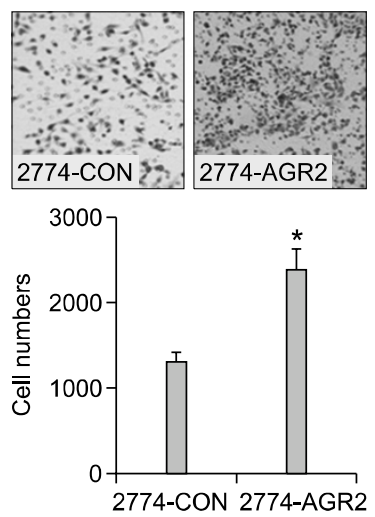

Figure 4. AGR2 overexpression increased cell growth and cell migration of human ovarian cancer cells. (A) Control vector- and AGR2-overexpressing stable 2774 cells were plated onto 96 -well sensor plates at $2.5 \times 10^{3}$ cells per well, then incubated overnight. Growth of cells was continuously monitored using real-time cell electronic sensing (RT-CES) system. (B) Enhancement of cancer cell migration by AGR2 overexpression. Cancer cell migration was assayed using Transwells after coating the lower surface of the filter with gelatin. The number of cancer cells that migrated to the lower side of the filter was counted. The results are the means $\pm \mathrm{SD}\left({ }^{*}, P<0.05\right.$ compared to $\left.2774-\mathrm{CON}\right)$.

serum of the mucinous-type ovarian cancer, as shown by Western blot analysis (top panel). Expression of AGR2 protein in patient serum was also analyzed by ELISA methods. Five mucinoustype ovarian cancer serum samples, 9 serous-type samples, and 11 normal healthy women samples were assessed for the detection of AGR2 by ELISA and summarized (Figure 5B). The elevated expression of serum AGR2 protein was detected in

mucinous ovarian tumor sera compared with normal and serous-type ovarian tumor sera by ELISA. The significance of the results was assessed by the Kruskal-Wallis rank-sum test $(P<0.0034)$.

\section{Discussion}

Cancer cells are known to acquire the hallmarks of malignancy through the accumulation of advantageous gene activation and inactivation events over long periods of time (Hanahan and Weinberg, 2000). To identify a novel ovarian tumor marker for early detection, pooled scrapings of human normal ovarian surface epithelium rather than whole ovaries were used to compare gene expression with ovarian tumors. Previously, AGR2 was reported to be expressed in cancers of the breast (Fritzsche et al., 2006), pancreas (Ramachandran et al., 2008; Riener et al., 2009), and prostate (Zhang et al., 2005). Overexpression or suppression of AGR2 was shown to affect cell proliferation, cell invasion, metastasis, and tumor growth of pancreatic cancer (Ramachandran et al., 2008). Recent reports support a role for AGR2 in cellular transformation and growth of esophageal adenocarcinoma (Wang et al., 2008).

Our systematic approach combining cDNA microarray analysis, RT-PCR, immunohistochemistry, Western blot analyses, and bioinformatics facilitated the identification of AGR2 as a novel candidate of an ovarian tumor marker for the early detection of mucinous ovarian tumors. More importantly, in terms of clinical usefulness, we were

Table 1. cDNA microarray analysis of AGR2 stable cells

\begin{tabular}{|c|c|c|c|c|c|c|c|}
\hline \multicolumn{8}{|c|}{ A. Genes up-regulated in AGR2 stable cells } \\
\hline GenBank & & Gene & \multicolumn{2}{|c|}{ Gene description } & $\begin{array}{l}\text { Normalized } \\
\text { ratio }\end{array}$ & $\begin{array}{l}\text { Subcellular } \\
\text { localization }\end{array}$ & Biological function \\
\hline NM_002961 & & S100A4 & \multicolumn{2}{|c|}{ S100 calcium binding protein A4 } & 2.43 & Cytoplasm & Invasive growth \\
\hline NM_005980 & & S100P & S100 calcium binding & $g$ protein $\mathrm{P}$ & 5.01 & Cytoplasm & Cell life, proliferation/growth \\
\hline NM_003225 & & TFF1 & Trefoil factor 1 & & 4.19 & Secreted & Cell growth and/or maintenance \\
\hline NM_0010071 & & IGF2 & Insulin-like growth fac & ctor 2 & 3.84 & Secreted & Cell growth \\
\hline NM_00102536 & & VEGFA & Vascular endothelial gr & rowth factor $A$ & 3.44 & Secreted & Angiogenesis \\
\hline NM_000029 & & $A G T$ & Angiotensinogen & & 4.45 & Secreted & Angiogenesis \\
\hline \multicolumn{8}{|c|}{ B. Genes down-regulated in AGR2 stable cells } \\
\hline GenBank & Gene & \multicolumn{2}{|r|}{ Gene description } & $\begin{array}{l}\text { Vormalized } \\
\text { ratio }\end{array}$ & $\begin{array}{l}\text { Subcellular } \\
\text { localization }\end{array}$ & & Biological function \\
\hline NM_001717 & $B N C 1$ & \multicolumn{2}{|c|}{ Basonuclin 1} & 0.25 & cleus & \multicolumn{2}{|c|}{ Regulation of transcription, DNA dependent } \\
\hline BC016333 & CCNL2 & \multicolumn{2}{|c|}{ Cyclin L2 } & 0.47 & cleus & \multicolumn{2}{|c|}{ Regulation of cell cycle } \\
\hline NM_001793 & $\mathrm{CDH} 3$ & \multicolumn{2}{|c|}{ Cadherin 3 , type 1 , P-cadherin } & 0.29 & egral to membr & \multicolumn{2}{|c|}{ Cell adhesion } \\
\hline NM_001425 & EMP3 & \multicolumn{2}{|c|}{ Epithelial membrane protein 3} & 0.39 & egral to membr & \multicolumn{2}{|c|}{ Cell death } \\
\hline NM_003246 & THBS1 & \multicolumn{2}{|c|}{ Thrombospondin 1} & 0.40 & tracellular matri & \multicolumn{2}{|c|}{ Cell adhesion } \\
\hline NM_000584 & IL8 & \multicolumn{2}{|c|}{ Interleukin 8} & 0.50 & tracellular spac & \multicolumn{2}{|c|}{ Negative regulation of cell proliferation } \\
\hline
\end{tabular}


A

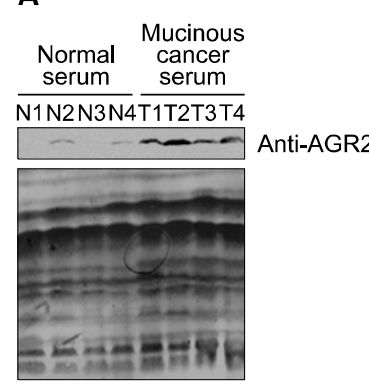

\section{B}

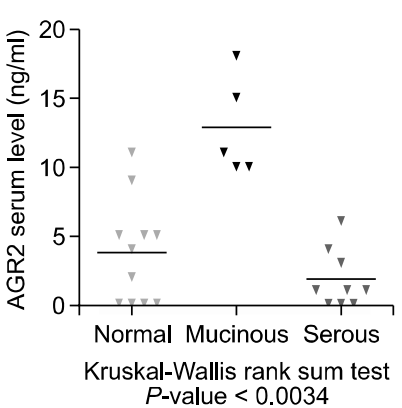

Figure 5. Detection of AGR2 protein in the serum from mucinous ovarian cancer patients. (A) Serum AGR2 level was assessed by Western blot analysis of de-albuminized sera derived from the normal volunteers and tumor patients after processing using immunoaffinity albumin and IgG depletion kit. Equal serum protein loading was confirmed by staining the same blot with Ponceau S. (B) Twenty-five sera (5 mucinous-type ovarian cancer, 9 serous-type ovarian cancer, and 11 normal ovarian samples) were processed for the detection of AGR2 protein by ELISA assay and summarized by scatter plot analysis. Statistical significance was analyzed by the Kruskal-Wallis rank sum test $(P<0.005)$.

able to detect AGR2 in patient serum by Western blot and ELISA assays. Detection in patient serum by an ELISA assay developed in this study provides an efficient screening of mucinous ovarian tumors among other ovarian tumors. Cumulative molecular genetic analyses of ovarian cancers have revealed genetic alterations of several genes in ovarian tumors, such as c-erb-B2, c-myc, and p53 (Aunoble et al., 2000). However, none of these mutations are diagnostic of malignancy. Previous reports by other investigators have identified multiple molecular targets that differ in normal and malignant cells in epithelial ovarian cancer, including cell cycle regulators, growth factor receptors, signal transduction pathways, genes that regulate drug resistance, and angiogenesis (reviewed in See et al., 2003; Sharma and Odunsi, 2005). In addition, AGR2 was shown to be a useful marker for detection of circulating tumor cells (CTCs) in peripheral blood of advanced cancer patients (Smirnov et al., 2005). The AGR2 protein has been previously associated with breast carcinomas by suppression subtractive cDNA library screening between a malignant human breast cancer cell line (MCF-7) and a benign breast tumor-derived cell line (Huma 123; Liu et al., 2005). Screening of breast tumor specimens using RTPCR and immunocytochemistry with anti-AGR2 antibodies has demonstrated that the presence of AGR2 mRNA and protein is statistically and significantly associated with ER $\alpha$-positive carcinomas and malignancy (Fritzsche et al., 2006). In addition, expression of AGR2 has been reported to be strongly associated with markers of tumor differentiation (estrogen receptor positivity and lower tumor grade). Although CA-125 is the best clinical marker for ovarian cancer in the postmenopausal age group, CA-125 has proven to be a poor diagnostic tumor biomarker for early stage ovarian cancer (Liede et al., 2002). Importantly, the CA-125 level was not elevated in the mucinous subtype. Previous efforts to characterize serum biomarkers for the detection of early-stage ovarian cancer identified transthyretin, beta-hemoglobin, apolipoprotein $\mathrm{A} 1$, and transferrin using micro-LC-MS/MS (Kozak et al., 2005). Unfortunately, the combination of CA-125 with biomarkers, such as transthyretin, beta-hemoglobin, apolipoprotein $A 1$, and transferrin, did not further improve the detection of mucinous tumors (Kozak et al., 2005). There has been an increase in the search for ovarian cancer markers using SELDI-TOF-MS with the use of multiple markers (Zhang et al., 2004), but there is still no significant improvement for detection of mucinous ovarian cancer. Considering the preferential expression of borderline mucinous cancer, AGR2 would be beneficial to improve the early detection of mucinous ovarian tumors.

To gain insight into the role of AGR2 in ovarian tumorigenesis, AGR2-overexpressing stable human ovarian cancer cells were generated and our results showed that AGR2 enhanced cell growth and migration of ovarian cancer cells. Consistent with the involvement of AGR2 in cell growth and migration, our cDNA microarray analysis of AGR2stable cells revealed that AGR2 overexpression up-regulated the expression of genes involved in cell proliferation, invasion, and angiogenesis, which is important for the tumor progression and metastasis characteristic of ovarian cancer. By contrast, the genes involved in the negative regulation of cell proliferation, cell adhesion, and cell death were down-regulated. Moreover, AGR2 protein can be detected in the serum of mucinous ovarian tumor patients by Western blot and ELISA methods. These results suggest that serum AGR2 can be a biomarker for diagnosis and prediction of mucinous ovarian cancers. This is the first report that AGR2 is a candidate molecular marker for mucinous ovarian tumors. However, validation of AGR2 as a potential serum marker is needed with a large-scale analysis of ovarian cancer blood samples and can be compared with other validated serum markers. We continue to expand our effort to collect serum from mucinous ovarian cancer patients and normal volunteers. Because AGR2 has previously been reported to be associated with tumor metastasis, it would be interesting to determine the role of AGR2 in ovarian tumor pro- 
gression and metastasis. Analysis of AGR2 protein suggests potential protein modification that might be important for AGR2 function (Park and Lee, unpublished data). Further functional analysis of AGR2 protein modification during tumor progression would provide information for its functional significance in addition to its role in diagnosis. Recently AGR2 was classified as a member of the protein disulfide isomerase (PDI) family and shown to play a special role in the production of mucus (Park et al., 2009). AGR2 is also expressed in mucus-producing cells, and AGR2-deficient mice were unable to produce intestinal mucin and became highly susceptible to experimentally-induced colitis.

The impact of results in this study on cancer biomarker discovery efforts is significant because ovarian cancer remains a highly fatal cancer due to the lack of reliable screening tests and high recurrence of ovarian cancer. The results from this study suggest that AGR2 can be a potential biomarker for diagnosis of mucinous ovarian cancer and the ELISA assay would facilitate the early detection of mucinous ovarian cancer using a non-invasive method using patient serum.

\section{Methods}

\section{Cell culture}

Human ovarian cancer (2774 cells) and human fetus kidney epithelial cells (HEK 293 cells) were cultured in DMEM and EMEM, respectively, and supplemented with $10 \%$ FBS and antibiotics (penicillin [100 units $/ \mathrm{ml}$ ] and streptomycin [100 $\mu \mathrm{g} / \mathrm{ml}]$; Invitrogen Corporation, Carlsbad, $\mathrm{CA}$ ) in a $5 \% \mathrm{CO}_{2}$ humidified atmosphere at $37^{\circ} \mathrm{C}$.

\section{Tissue and serum protein preparation}

All human tissue and serum samples were collected with institutional approval at Samsung Medical Center. All ovarian tissues, including normal and tumor sources, were collected from women who underwent surgery in the Department of Obstetrics and Gynecology at Samsung Medical Center. The tissue samples were immediately stored in liquid nitrogen and stored at $-80^{\circ} \mathrm{C}$ until analysis. Paraffin archives from the Department of Pathology of Samsung Medical Center were used to confirm the expression of AGR2 by immunohistochemistry. All serum samples were collected by venipuncture from patients and healthy donors. Sera from women with ovarian cancer were collected before surgical removal of the ovaries. Both the stage and histology of the cancer were determined by standard pathologic methods. Serum was separated, centrifuged $(3000 \times \mathrm{g}, 20 \mathrm{~min})$, and stored at $-80^{\circ} \mathrm{C}$ in 50 $\mu \mathrm{l}$ aliquots until use. Serum specimens were used to detect the serum AGR2 protein level by Western blot analysis after depleting albumin and IgG from serum samples using a Vivapure anti-HAS/lgG kit (Sartorius Stedim Biotech,
Bohemia, NY) according to the manufacturer's protocol.

\section{Chemicals}

Antibodies against AGR2 were purchased from Abnova (clone 1C3; Taipei city, Taiwan) and Abcam (rabbit polyclonal; Cambridge, UK). Tubulin and lamin B were purchased from Santa Cruz Biotechnology, Inc. (Santa Cruz, $\mathrm{CA}$ ), and $\beta$-actin from Sigma (St. Louis, MO). 4',6-diamidio-2-phenylindole (DAPI) was purchased from Roche Applied Science (Mannheim, Germany). All other chemical reagents were purchased from Sigma, unless otherwise indicated.

\section{Stable transfection and generation of AGR2-overexpressing human ovarian cells}

Human 2774 ovarian cancer cells were transiently transfected with control vector or AGR2-expressing vector using Effectene (Qiagen, Hilden, Germany) according to the manufacturer's instructions. In brief, 2774 cells were seeded the day before transfection, then incubated until reaching $60 \%$ confluence on the day of transfection. After selection in medium containing G418 (500 $\mu \mathrm{g} / \mathrm{mL}$; Invtrogen), G418resistant discrete colonies were selected for further expansion in selection medium. Several clones for vector control and AGR2-overexpressing 2774 cells were maintained with DMEM containing G418.

\section{RNA isolation, CDNA microarray analysis, and RT-PCR}

Total cellular RNA was extracted from ovarian tumor and matched normal tissues obtained from 32 patients using TRIZOL reagent (Invitrogen Life Technologies), according to the manufacturer's protocol. Human 17K cDNA microarrays (Genomictree, Daejeon, Korea) containing 17,075 human cDNAs representing 15,724 different genes (UniGene clusters) were used in gene expression analysis, as previously described (Yang et al., 2003). Briefly, each 50 $\mu \mathrm{g}$ of total RNA was mixed with $2 \mu \mathrm{g}$ of oligo-(dT) 24 (GenoTech, Daejeon, Korea) and incubated at $65^{\circ} \mathrm{C}$ for 10 min. Single-stranded cDNA was synthesized in the presence of Cy3-dUTP or Cy5-dUTP (1 mM each, NEN Life Science Products, Boston, MA) at $42^{\circ} \mathrm{C}$ for $2 \mathrm{~h}$. Total RNAs from ovarian tumors and normal ovary tissues were labeled with Cy5 and Сy3, respectively. Both Cy5- and Cy3-labeled cDNA were purified using PCR purification kit (Qiagen) and hybridized onto human $17 \mathrm{~K}$ cDNA microarrays. The arrays were incubated at $42^{\circ} \mathrm{C}$ for $16 \mathrm{~h}$ in the humidified hybridization chamber. The hybridized microarrays were imaged by using the Axon 4000B scanner (Molecular Devices, CA). The signals and background fluorescence intensities were calculated for each probe by averaging the intensities of every pixel inside probe spot region using GenePix Pro 4.0 software (Molecular Devices). Spots of poor quality or indistinguishable signal levels from the background were excluded. Data normalization and selection of differentially expressed genes were performed using GeneSpring 7.3.1 (Agilent Technologies, Palo Alto, CA). To analyze differentially expressed 
genes between tumor and paired normal ovary tissues, the "Cross gene error model for replicates" was used. Gene expression data was normalized using global LOWESS normalization. Probes which were changed $\geq 2$.0-fold ratio between tumor and control normal ovary tissues were selected and considered as differentially-expressed probes. For RT-PCR analysis, $1 \mu \mathrm{g}$ of total RNA was dissolved in distilled water, then used for cDNA synthesis by superscript reverse transcriptase (RT; Invitrogen), then PCR amplification was carried out with specific AGR2 primers as follows: $94^{\circ} \mathrm{C}$ for $3 \mathrm{~min}$, followed by 20 cycles at $94^{\circ} \mathrm{C}$ for $30 \mathrm{~s}, 55^{\circ} \mathrm{C}$ for $30 \mathrm{~s}$, and $72^{\circ} \mathrm{C}$ for $1.5 \mathrm{~min}$. The following primers were used for PCR amplification: forward primer, 5'-GGGATGGAGAAAATTCCAGTG-3'; and reverse primer, 5'-GGGTACAATTCAGTCTTCAG-3'. PCR products were separated by $1.2 \%$ agarose gel electrophoresis. GAPDH was used as a loading control.

\section{Subcellular fractionation}

Subcellular fractionation was carried out using a ProteoExtract Subcellular Proteome Extraction kit (Calbiochem, Darmstadt, Germany). Cultured cells were harvested with $500 \mu \mathrm{l}$ of cell lysis buffer $(10 \mathrm{mM}$ Tris-HCl $[\mathrm{pH}$ 7.5], $10 \mathrm{mM} \mathrm{NaCl}, 1 \mathrm{mM} \mathrm{KH}_{2} \mathrm{PO}_{4}, 5 \mathrm{mM} \mathrm{NaHCO} 3,1 \mathrm{mM}$ $\mathrm{CaCl}_{2}$, and $0.5 \mathrm{mM} \mathrm{MgCl}$ ) with a protease inhibitor cocktail (Roche Diagnostics Corp. Penzberg, Germany). Cells were allowed to swell for $5 \mathrm{~min}$, then homogenized 50 times using a Dounce homogenizer (Wheaton, Millville, IL). Lysates were centrifuged for $10 \mathrm{~min}$ at $7,500 \mathrm{rpm}$ in a centrifuge at $4^{\circ} \mathrm{C}$, and the cytoplasmic fraction was transferred to a clean tube. The nuclear pellet was washed once with cell lysis buffer before resuspending with SDS-loading buffer. The efficiency of subcellular fractionation was confirmed by Western blot analysis with anti-lamin B and anti-tubulin as nuclear and cytoplasmic protein marker antibodies, respectively.

\section{Western blot analysis}

Cultured cells and tissue lysates were lysed by sonication in phosphate-buffered saline with RIPA buffer $(50 \mathrm{mM}$ Tris- $\mathrm{HCl}$ [pH 7.5], $150 \mathrm{mM} \mathrm{NaCl}, 1 \% \mathrm{NP} 40,0.5 \%$ sodium deoxycholate, and $0.1 \%$ SDS) containing protease inhibitors (protease inhibitor mixture; Roche Applied Science), as described previously (Park et al., 2005). Proteins were separated by SDS-PAGE and electrophoretically transferred onto an ECL nitrocellulose membrane (GE Healthcare, London, UK). Western blot analysis was performed with the primary antibody (1:1000 or 1:2000 dilution) and an enhanced chemiluminescence system (ECL; Amersham Sciences, Chicago, IL).

\section{Indirect immunofluorescence staining and confocal microscopy}

Cells were grown on sterilized glass coverslips, fixed with $4 \%$ paraformaldehyde in $0.1 \mathrm{M}$ phosphate buffer for 15 min, then blocked with $0.1 \%$ BSA in PBS. Then cells were incubated with anti-AGR2 (1:500 dilution) in PBS, followed by incubation with Alexa 488-conjugated secondary antibody (1:5000 dilution; Vector, Burlingame, CA). The slides were washed three times with PBS, and mounted in mounting media (Vectorshield; Vector). Images were captured with a Bio-Rad confocal microscope (Bio-Rad Laboratories, Hercules, CA).

\section{Immunohistochemistry}

Immunohistochemical analysis was carried out on a formalin-fixed, paraffin-embedded specimen cut at a thickness of $4 \mu \mathrm{m}$. The paraffin block specimens used in the current experiments were derived from archived tissue (not representing samples from patients who contributed fresh specimens) derived from patients having surgery at the Samsung Medical Center. All slides were deparaffinized in xylene, rehydrated through a graded alcohol series, and washed in PBS. The slides were treated with $0.3 \%$ hydrogen peroxide in PBS for $5 \mathrm{~min}$ at room temperature to quench the endogenous peroxidase activity. For antigen retrieval, sections were microwaved twice for $10 \mathrm{~min}$ in 10 $\mathrm{mM}$ citrate buffer ( $\mathrm{pH}$ 6.0). For AGR2 staining, polyclonal rabbit antisera against peptides derived from the AGR2 protein sequence (dilution 1:250; Abcam) were used and the primary antibody was incubated at $4^{\circ} \mathrm{C}$ overnight. As a negative control, slides were also processed without primary antibody. Detection was done by the conventional streptavidin-biotin method with 3,3-diaminobenzidine tetrahydrochloride as a chromogen (DAKO, Copenhagen, Denmark). The slides were counterstained with hematoxylin for $5 \mathrm{~min}$, then dehydrated by sequentially washing in an ethanol series and rinsing three times with xylene. The slides were then mounted with Consul-mount (Thermo Scientific, Waltham, MA).

\section{Subcloning of human AGR2}

The full-length coding sequence of human AGR2 was PCR-amplified using AGR2 primers, as described below. Thermal cycling conditions comprised an initial step at $95^{\circ} \mathrm{C}$ for $5 \mathrm{~min}$, followed by 20 cycles at $95^{\circ} \mathrm{C}$ for $1 \mathrm{~min}$, $55^{\circ} \mathrm{C}$ for $1 \mathrm{~min}, 72^{\circ} \mathrm{C}$ for $2 \mathrm{~min}$, then a final extension at $72^{\circ} \mathrm{C}$. The forward primer was $5^{\prime}$-GGGGAATTCATGGAGAAAATTCCAGTG-3' and the reverse primer was 5'-GGGCTCGAGTTACAATTCAGTCTTGAG-3'. Amplified full-length AGR2 was then subcloned into EcoR1-Xho l-digested mammalian expression vector pcDNA3.1 Myc-His (Invitrogen). The sequence of AGR2 in mammalian vector was confirmed by DNA sequence analysis.

\section{Real-time cell growth monitoring}

The effect of AGR2 overexpression on cell growth was assessed using the real time electronic sensing system (RT-CES; ACEA Biosciences, San Diego, CA), according to the manufacturer's protocol. Control vector- and AGR2overexpressing stable 2774 cells were plated onto 96-well sensor plates at $2.5 \times 10^{3}$ cells per well, then incubated overnight. Cells were continuously monitored using a RTCES.

\section{In vitro migration assays}

Migration of ovarian cancer cells was assessed using a 
Biocoat matrigel chamber (Becton Dickinson Labware, Bedford, MA), as previously described (Choi et al., 2005). Briefly, $1 \times 10^{5}$ cells were plated in each well and the bottom chamber outside the transwell was filled with $500 \mu \mathrm{l}$ of DMEM medium supplemented with $1 \%$ BSA. After incubation for $16 \mathrm{~h}$, the cells were fixed in $100 \%$ methanol for $5 \mathrm{~min}$ and stained in $0.1 \%$ eosin. The number of cells that migrated to the lower side of the filter was counted.

\section{Sandwich ELISA assay}

To develop a sensitive sandwich ELISA assay for AGR2 protein detection in patient serum, optimized dilutions of monoclonal AGR2 antibodies were coated onto 96-well Immobilon plates overnight at $4^{\circ} \mathrm{C}$ in $1 \times$ PBS buffer. After washing, plates were blocked with $0.05 \%$ Tween $20 / 2.5 \%$ $\mathrm{BSA} / 1 \times \mathrm{PBS}$ at RT for $1 \mathrm{~h}$. Patient serum was added to the well, then incubated for $2 \mathrm{~h}$ at RT. After washing unbound antigen solutions, the second anti-AGR2 antibody was added and incubated for $4 \mathrm{~h}$. After washing unbound antibody, HRP-conjugated goat anti-mouse IgG was added at a 1:5000 dilution. Detection was performed with Super AquaBlue ELISA substrate (eBioscience, San Diego, CA) and stopped with $0.5 \mathrm{M}$ sulfuric acid. Plates were read in a Tunable microplate reader (Molecular Devices) at $405 \mathrm{~nm}$ and analysis was performed using the SoftMax Pro v4.3 LS software (Molecular Devices).

\section{Statistical analysis}

Experiments were independently performed three or more times. The data are presented as the mean \pm standard deviation. An unpaired Student's $t$-test was used to compare the means of cell migration between the vector control and AGR2-overexpressing 2774 stable cells. The Kruskal-Wallis rank-sum test was used to evaluate the significant difference in AGR2 serum levels between normal volunteers and ovarian cancer patients. $P$-values $<0.05$ were considered statistically significant.

\section{Acknowledgements}

We thank Charles Y.F. Young for providing anti-AGR2 antibody for the initial study of AGR2 protein expression. We also thank $\mathrm{YH}$ Kim for reading the manuscript. This study was supported by Samsung Biomedical Research Institute grant (C-A9-232), a SRC grant from KOSEF (R1102000-080-11001), a grant from the Korea Health Planning Technology and Evaluation Board (A090918), and a grant from the National R\&D Program for Cancer Control (0920010), Ministry for Health, Welfare and Family Affairs, Republic of Korea.

\section{References}

Aberger F, Weidinger G, Grunz H, Richter K. Anterior specification of embryonic ectoderm: the role of the Xenopus cement gland-specific gene XAG-2. Mech Dev 1998;72: $115-30$

American Cancer Society. Cancer facts and figures 2005 [online]. Atlanta, GA, available from: URL: http://www.cancer. org/docroot/stt/stt_0.asp

Aunoble B, Sanches R, Didier E, Bignon YJ. Major oncogenes and tumor suppressor genes involved in epithelial ovarian cancer (review). Int J Oncol 2000;16:567-76

Choi J, Choi K, Benveniste EN, Rho SB, Hong YS, Lee JH, Kim J, Park K. Bcl-2 promotes invasion and lung metastasis by inducing matrix metalloproteinase-2. Cancer Res 2005; 65:5554-60

Edwards BK, Brown ML, Wingo PA, Howe HL, Ward E, Ries LA, Schrag D, Jamison PM, Jemal A, Wu XC, et al. Annual report to the nation on the status of cancer, 1975-2002, featuring population-based trends in cancer treatment. J Natl Cancer Inst 2005;97:1407-27

Fletcher GC, Patel S, Tyson K, Adam PJ, Schenker M, Loader JA, Daviet L, Legrain P, Parekh R, Harris AL, et al. hAG-2 and hAG-3, human homologues of genes involved in differentiation, are associated with oestrogen receptorpositive breast tumours and interact with metastasis gene C4.4a and dystroglycan. Br J Cancer 2003;88:579-85

Fritzsche FR, Dahl E, Pahl S, Burkhardt M, Luo J, Mayordomo E, Gansukh T, Dankof A, Knuechel R, Denkert C, et al. Prognostic relevance of AGR2 expression in breast cancer. Clin Cancer Res 2006;12:1728-34

Hanahan D, Weinberg RA. The hallmarks of cancer. Cell 2000;100:57-70

Komiya T, Tanigawa Y, Hirohashi S. Cloning of the gene gob-4, which is expressed in intestinal goblet cells in mice. Biochim Biophys Acta 1999;1444:434-8

Kozak KR, Su F, Whitelegge JP, Faull K, Reddy S, Farias-Eisner R. Characterization of serum biomarkers for detection of early stage ovarian cancer. Proteomics 2005; 5:4589-96

Kristiansen G, Pilarsky C, Wissmann C, Kaiser S, Bruemmendorf T, Roepcke S, Dahl E, Hinzmann B, Specht T, Pervan $\mathrm{J}$, et al. Expression profiling of microdissected matched prostate cancer samples reveals CD166/MEMD and CD24 as new prognostic markers for patient survival. J Pathol 2005;205:359-76

Liede A, Karlan BY, Baldwin RL, Platt LD, Kuperstein G, Narod SA. Cancer incidence in a population of Jewish women at risk of ovarian cancer. J Clin Oncol 2002;20:1570-7

Liu D, Rudland PS, Sibson DR, Platt-Higgins A, Barraclough, R. Human homologue of cement gland protein, a novel metastasis inducer associated with breast carcinomas. Cancer Res 2005;65:3796-805

Park K, Kim K, Rho SB, Choi K, Kim D, Oh SH, Park J, Lee $\mathrm{SH}$, Lee JH. Homeobox Msx1 interacts with p53 tumor suppressor and inhibits tumor growth by inducing apoptosis. Cancer Res 2005;65:749-57

Park K, Choi K, Kim H, Kim K, Lee MH, Lee JH, Kim Rim JC. Isoflavone-deprived soy peptide suppresses mammary tumorigenesis by inducing apoptosis. Exp Mol Med 2009; 41:371-81

Park SW, Zhen G, Verhaeghe C, Nakagami Y, Nguyenvu LT, Barczak AJ, Killeen N, Erle DJ. The protein disulfide iso- 
merase AGR2 is essential for production of intestinal mucus. Proc Natl Acad Sci USA 2009;106:6950-5

Ramachandran V, Arumugam T, Wang $\mathrm{H}$, Logsdon CD. Anterior gradient 2 is expressed and secreted during the development of pancreatic cancer and promotes cancer cell survival. Cancer Res 2008;68:7811-8

Riener MO, Pilarsky C, Gerhardt J, Grutzmann R, Fritzsche FR, Bahra M, Weichert W, Kristiansen G. Prognostic significance of AGR2 in pancreatic ductal adenocarcinoma. Histol Histopathol 2009;24:1121-8

See HT, Kavanagh JJ, Hu W, Bast RC. Targeted therapy for epithelial ovarian cancer: current status and future prospects. Int J Gynecol Cancer 2003;13:701-34

Sharma S, Odunsi K. Targeted therapy for epithelial ovarian cancer. Expert Opin Ther Targets 2005;9:501-13

Smirnov DA, Zweitzig DR, Foulk BW, Miller MC, Doyle GV, Pienta KJ, Meropol NJ, Weiner LM, Cohen SJ, Moreno JG, et al. Global gene expression profiling of circulating tumor cells. Cancer Res 2005;65:4993-7

Wang Z, Hao Y, Lowe AW. The adenocarcinoma-associated antigen, AGR2, promotes tumor growth, cell migration, and cellular transformation. Cancer Res 2008;68:492-7

Yang SH, Kim JS, Oh TJ, Kim MS, Lee SW, Woo SK, Cho HS, Choi YH, Kim YH, Rha SY, Chung HC, An SW. Genome-scale analysis of resveratrol-induced gene expression profile in human ovarian cancer cells using a cDNA microarray. Int $\mathrm{J}$ Oncol 2003;22:741-50

Zhang JS, Gong A, Cheville JC, Smith DI, Young CY. AGR2, an androgen-inducible secretory protein overexpressed in prostate cancer. Genes Chromosomes Cancer 2005;43: 249-59

Zhang Z, Bast RC Jr, Yu Y, Li J, Sokoll LJ, Rai AJ, Rosenzweig JM, Cameron B, Wang YY, Meng XY, et al. Three biomarkers identified from serum proteomic analysis for the detection of early stage ovarian cancer. Cancer Res 2004;64:5882-90 